\title{
Survey of Jordanian Awareness about Hazardous Symbols of Chemicals
}

\author{
Nawal Bahtiti*a, Ahmad Abu Rayyan ${ }^{\mathrm{B}}$, Tala SaSA ${ }^{\mathrm{A}}$, Waed Alahmad ${ }^{\mathrm{A}}$ \\ ${ }^{a}$ Basic Science Department, Faculty of Science, Applied Science Private University, P.O. Box 166 \\ Amman 11931, JORDAN \\ ${ }^{\mathrm{b}}$ Chemistry Department, Faculty of Science, Applied Science Private University, P.O. Box 166 \\ Amman 11931, JORDAN
}

\begin{abstract}
The use of chemical substances has commonly increased, there are such a number of chemical dangers all spherical us that it is probably almost now no longer feasible to feature if we centered constantly on the dangers. This is precisely why we need to don't forget the dangers. Everyone need to apprehend exactly what do in case of unstable contact with risky material. Previously we tested consciousness of Jordanian

peoples and measured the employees' interest of risky chemical compounds1. So this new seek aimed to research chemical symbols attentions, a questionnaire survey come to be executed among a whole of 245 peoples. The questionnaire come to be acquainted with flammable risky symbols as $90.6 \%$, however handiest $7 \%$ for fitness chance symbol. Statistical assessment of the statistics come to be finished with the Statistical Package for Social Sciences (SPSS) version 25. The effects show that the descriptive information confirmed that scholars proven truthful to excellent familiarity and expertise of chemical chance caution symbols. Most college students had bad to truthful attitudes closer to chemical laboratory protection; however, the evaluation of college students' chemical laboratory protection practices found out truthful to suitable practices. While college sfudents' protection focus and practices, howeyer now no longer attitude at this collegg haye been
acceptable, protection tactics want to be applied inside an extra expert protectioft thainfifg aqd and protection weather management.
\end{abstract}

Key-Words: - Chemical hazard; Awareness; Jordanian, Employees, Risk, Dangerous symbols.

Received: March 2, 2021. Revised: September 14, 2021. Accepted: September 23, 2021. Published: October 1, 2021.

\section{Introduction}

Many chemical compounds which are being produced and utilized in merchandise, substances and homes are dangerous for human fitness and the surroundings (1). There are legal guidelines to make chemical utilization more secure and to defend human fitness and the surroundings. Despite efforts on a structural degree human beings are nonetheless being uncovered via touch with merchandise that include dangerous chemical compounds or via dust, indoor-air, water and meals in addition to pores and skin absorption Modern society is experiencing a length of exceptional intake with an amazing multitude of chemical materials being utilized in patron articles and industrial mixtures. Many materials labeled as dangerous in keeping with the EU law on type and labeling (CLP Regulation) 1 are found in regular merchandise as everyday ingredients, like, for example, preservatives in washing and cleansing agents, fragrances in nonpublic care merchandise, per- and polyfluorinated chemical compounds utilized in fabric finishing, plasticizers in plastic substances, or heavy metals in digital appliances. Many of those materials stay left out via way of means of the common end-consumer who takes the blessings of the chemical ingredients as a right and trusts that undesirable residences for guy and the surroundings are negligible. Risk communication provisions, such as hazard pictograms on the product containers, are established to aid consumers and workers to be aware of hazards and to implement a suitable risk management behavior so as to minimize exposure and hence risk. The understanding of the risk communication message by the recipients is one of the basic pillars of chemical legislation2. Risk communication is an important first step, but there are various indications that risk communication measures are not always as effective as intended, because they are not always understood in the way expected by the decision makers and are thus not sufficiently protective under the consumer and the environmental perspectives. Previous studies that evaluated the efficiency and effectiveness of risk 
communication yielded remarkable results: A large number of users in European and non-European countries struggled to understand ingredient lists and labels 3,4. Other studies analyzed the understanding of hazard pictograms and showed that end-users did not understand the signs correctly 5,6. Even correctly understood risk information did not necessarily lead to the intended risk reduction behaviors 5 . It was also described that illiterate persons had great difficulty to understand pictorial label information and safety instructions 6. A European survey 7 where citizens should indicate whether they thought that certain products contained 'chemical substances' in general showed that it is also worthwhile to ask very simple and basic questions which do not require any previous knowledge. Participants of this survey were not asked about harmful substances but only about chemical ingredients 8,9 . For a chemist the results of this survey were shocking because large numbers of participants in Jordan did not know chemical hazards symbols.10.11 Such results suggest that these citizens might have problems understanding risk communication tools. However, it is not certain that experts judge risks correctly.

\section{Methods}

\subsection{Study Design}

The survey was conducted between September and December 2020 among Bachelors chemistry graduated in Jordan in several working sectors (teaching, Laboratory work and in manufactures, and others).

\subsection{Survey Instruments}

The questionnaire was developed based on the literature review of comparable studies. The questionnaire consisted of 36 items revealed: Assessment of Familiarity and Understanding of Chemical Hazard Warning Signs, Most abundant chemical in our life their presence and health effect.

\section{Statistical Analysis}

Statistical analysis was developed using STATA software program, version 16 (Stata Corporation. College Station, Tx). Data were summarized using frequencies and percentages for categorical data and mean and standard deviations for continuous data. Univariate and stepwise multivariate logistic regression analyses were performed to determine the independent association of explanatory variables with the following outcomes of interest: and research fellows, and $25.5 \%$ had been working in the current lab for less than 1 year; overall, more than half reported at least one working experience in other labs. About half of the workers reported to have a chronic illness and $67.9 \%$ had attended a GP in the previous year (Table 1).

\section{Conclusion}

According to table 3 . There is significant difference between the answers of male and female about (Corrosion) $q 5 \mathrm{p}=0.005<0.05$. The percentage of correct answers with respect to female is more than the percentage of correct answer with respect to male.

According to table 4. There is significant difference between the answers with respect to age about (flammable) q $2 \mathrm{p}=0.006<0.05$, The highest percentage of correct answers of age 2330 , the lowest of age greater than 40 .

According of table 5. There is significant difference between the answers with respect to education qualification.

$\mathrm{q} 2$ (flammable) $\mathrm{p}=0.001<0.05$. The highest percentage of correct answers is in favour of current undergraduate students and laboratory technicians, the lowest of lab managers.

There is significant difference between the answers with respect to education qualification $\mathrm{q} 3$ (oxidizing) $\mathrm{p}=0.038<0.05$. The highest percentage of correct answers is in favour of current undergraduate students, the lowest of post graduate students.

There is significant difference between the answers with respect to education qualification q4 (gas cylinder $\mathrm{p}=0.022<0.05$. The highest percentage of correct answers is in favour of lab manager, the lowest of master or doctors or others.

\section{Conclusions}

Misconceptions approximately dangerous materials in merchandise may be risky for the non-public fitness and the environment. The survey shows that motivation, instructional level, and chemical understanding do now no longer robotically offer the suitable expertise of dangerous materials in merchandise. If well-knowledgeable customers aren't sufficiently successful to apply chance statistics factors as found out on this study, then this can be even greater the case for the overall public. 
Consumer recognition ought to be stipulated via way of means of a stepped forward statistics approach approximately chemical dangers in patron merchandise with an in depth participation of the goal companies and via way of means of greater efforts via way of means of government and manufactures to construct consider and to offer effortlessly comprehensible statistics.

We recommended to enhance the lifestyle of protection ethics and threat control a few of the college body of workers and college students who've more than one chemistry laboratories of their examine plan; specifically, the pharmaceutical and chemical engineering and biomedical engineering college students. This may be carried out with the aid of using organizing an Environmental Health and Safety Office on the college this is answerable for making use of and following up on compliance with protection regulations and procedures, and growing a direction on dangerous waste and threat control, to be made obligatory for all college students who're assignment an application of examine that entails chemical laboratory exercises.

\section{Acknowledgement:}

The authors acknowledge Applied Science Private University, Amman, Jordan, for the full financial support granted of this research article. Sincere thanks to all my Colleagues at basic science department, for creating inspiring conditions for work.

\section{References:}

[1] Waed R Al ahmad, Tala H. Sasa, Nawal H Bahtiti, Ahmed Abu-Rayan, Indian Journal of Forensic Medicine \& Toxicology Accepted (27-4-2021)

[2] Regulation (EC) No 1272/2008 of the European parliament and of the council of 16 December 2008 on classification, labelling and packaging of substances and mixtures. http://eur-

lex.europa.eu/LexUriServ/LexUriServ.do?uri= CELEX:32008R1272:en:NOT. Accessed 2 Aug 2017

[3] ECHA. Communication on the Safe Use of Chemicals: Study on the Communication of Information to the General Public (2012). ECHA-12-A-01-EN. https://echa.europa.eu//communication-on-the-safe-use-of-chemicals. Accessed 2 Aug 2017
[4] Riley DM, Fischhoff B, Small MJ, Fischbeck P. Risk Anal. 2001, 21(2),357

[5] Park K. Park's textbook of preventive and social medicine. 20 ed. Jabalpur, India: Banarasidas Bhanot Publishers; 2007. Chapter 16, Occupational Health; pp 658.

[6] Antonino JM. Health effects of welding. Crit Rev Toxicol 2003, 33,61.

[7] Lehtinen S, Rantanen J, Elgstrand K, Liesivuori J, Peurala M, Finnish Institute of Occupational Health, 2005,37,51.

[8] International Labour Organization (ILO). International classification of occupations 1968. Revised edn. Geneva: International Labour Office. http://www.ilo.org/public/libdoc/ilo/1969/69B0 9_35_engl.pdf (accessed 5 Jul 2011).

[9] Antonino JM. Crit Rev Toxicol 2003, 33, 61

[10] Almuhur, E., Al-Labadi, M., Shatarah, A., Khan, N. and Bashir, R. (2021), "Studying structure of Corona viridians, analyzing their geometry and focusing on the role of electronic applications in health awareness of viruses", International Journal of Human Rights in Healthcare, Vol. ahead-of-print No. ahead-ofprint. https://doi.org/10.1108/IJHRH-01-20210003

[11] Waed R Alahmad*a, Tala H. Sasa a, Nawal H Bahtiti a, Rula F Khuzai a and Sura I Al ayed b. Manage Awareness Attitude Anxiety experience and E-learning during COVID-19 pandemic evidence from ASU university 2021.Accepted at Indian journal of Forensic Medicine and Toxicology.

Appendix:

Table1. A hazard Warning Symbol

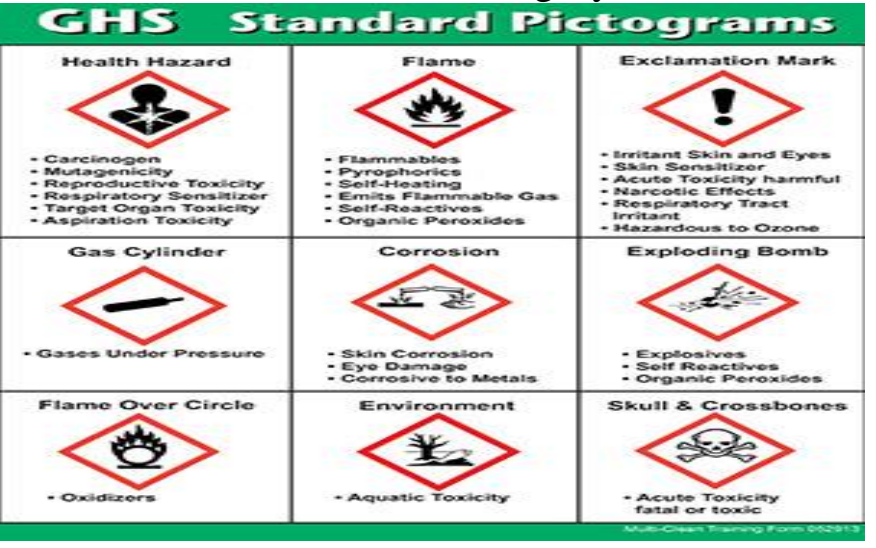


Table 2. Demographic, professional and knowledge of chemical hazards characteristics of the responders

\begin{tabular}{|c|c|c|c|}
\hline \multicolumn{2}{|l|}{ Characteristic } & Frequency & Percent \\
\hline \multirow{2}{*}{ Gender } & Male & 71 & 29.0 \\
\hline & Female & 174 & 71.0 \\
\hline \multirow{4}{*}{ Age } & $18-22$ & 154 & 62.9 \\
\hline & $23-30$ & 44 & 18.0 \\
\hline & $31-39$ & 15 & 6.1 \\
\hline & ABOVE 40 & 32 & 13.1 \\
\hline \multirow{7}{*}{ educational qualification } & Currently student & 160 & 65.3 \\
\hline & Post graduate students & 19 & 7.8 \\
\hline & Teachers & 14 & 5.7 \\
\hline & Lab technicians & 12 & 4.9 \\
\hline & Lab managers & 6 & 2.4 \\
\hline & $\begin{array}{l}\text { Instructors (master or } \\
\text { doctor) }\end{array}$ & 15 & 6.1 \\
\hline & Others & 19 & 7.8 \\
\hline \multirow{3}{*}{ Q1 Explosive symbol(1) } & Correct & 126 & 51.4 \\
\hline & Incorrect & 70 & 28.6 \\
\hline & Do not know & 49 & 20 \\
\hline \multirow{3}{*}{$\begin{array}{l}\text { Q2 Flammable symbol(2) } \\
\text { a }\end{array}$} & Correct & 222 & 90.6 \\
\hline & Incorrect & 18 & 7.3 \\
\hline & Do not know & 5 & 2 \\
\hline \multirow{3}{*}{$\begin{array}{l}\text { Q3 Oxidizer symbol (3) } \\
\text { (2) }\end{array}$} & Correct & 96 & 39.2 \\
\hline & Incorrect & 118 & 48.2 \\
\hline & Do not know & 31 & 12.7 \\
\hline \multirow{3}{*}{ Q4 Gas cylinder symbol (4) } & Correct & 117 & $47.8 \%$ \\
\hline & Incorrect & 79 & $32.2 \%$ \\
\hline & Do not know & 49 & $20 \%$ \\
\hline \multirow{3}{*}{ Q5 Corrosive symbol (5) } & Correct & 103 & $42 \%$ \\
\hline & Incorrect & 74 & $30.2 \%$ \\
\hline & Do not know & 68 & 27.8 \\
\hline \multirow{3}{*}{ Q6 Harmful symbol (6) } & Correct & 127 & $51.8 \%$ \\
\hline & Incorrect & 107 & $43.7 \%$ \\
\hline & Do not know & 11 & $4.5 \%$ \\
\hline \multirow{3}{*}{$\begin{array}{l}\text { Q7 Exclamation mark } \\
\text { symbol (7) } \\
\vdots\end{array}$} & Correct & 60 & $24.5 \%$ \\
\hline & Incorrect & 116 & $47.3 \%$ \\
\hline & Do not know & 69 & $28.2 \%$ \\
\hline \multirow{3}{*}{$\begin{array}{l}\text { Q8 Health hazard symbol } \\
\text { (8) }\end{array}$} & Correct & 20 & $8.2 \%$ \\
\hline & Incorrect & 194 & $79.2 \%$ \\
\hline & Do not know & 31 & $12.7 \%$ \\
\hline
\end{tabular}




\begin{tabular}{|c|c|c|c|}
\hline \multirow{3}{*}{$\begin{array}{l}\text { Q9 Environment hazard } \\
\text { symbol (9) }\end{array}$} & Correct & 181 & $73.9 \%$ \\
\hline & Incorrect & 23 & $9.4 \%$ \\
\hline & Do not know & 41 & $16.7 \%$ \\
\hline
\end{tabular}

Table 3. Percentage of correct answers, incorrect answer and not knowing the answer for respondents with respect to gender

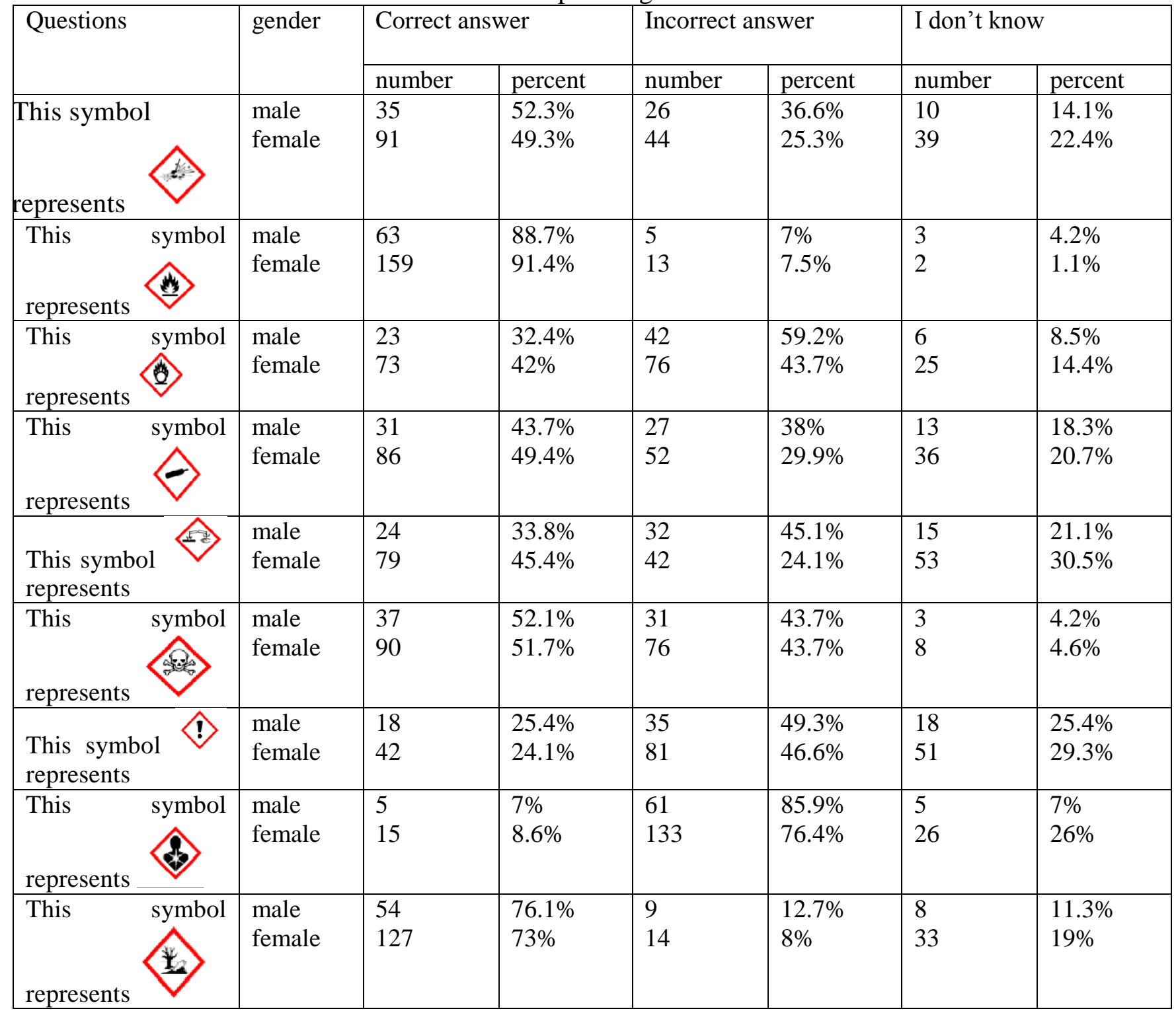

Table 4. Percentage of correct answers, incorrect answer and not knowing the answer for respondents with respect to age

\begin{tabular}{|l|l|l|l|l|}
\hline Questions & age & Correct answer & Incorrect answer & I don't know \\
\hline
\end{tabular}




\begin{tabular}{|c|c|c|c|c|c|c|c|}
\hline & & number & percent & number & percent & number & percent \\
\hline This symbol & $\begin{array}{l}18-22 \\
23-30 \\
31-39 \\
\text { Greater } \\
\text { than } 40\end{array}$ & $\begin{array}{l}81 \\
20 \\
9 \\
16\end{array}$ & $\begin{array}{l}52.6 \% \\
45.5 \% \\
60 \% \\
50 \%\end{array}$ & $\begin{array}{l}50 \\
13 \\
2 \\
5\end{array}$ & $\begin{array}{l}32.5 \% \\
29.5 \% \\
13.3 \% \\
15.6 \%\end{array}$ & $\begin{array}{l}23 \\
11 \\
4 \\
11\end{array}$ & $\begin{array}{l}14.9 \% \\
25 \% \\
26.7 \% \\
34.4 \%\end{array}$ \\
\hline This symbol & $\begin{array}{l}18-22 \\
23-30 \\
31-39 \\
\text { Greater } \\
\text { than } 40\end{array}$ & $\begin{array}{l}143 \\
43 \\
13 \\
23\end{array}$ & $\begin{array}{l}92.9 \% \\
97.7 \% \\
86.7 \% \\
71.9 \%\end{array}$ & $\begin{array}{l}8 \\
1 \\
2 \\
7\end{array}$ & $\begin{array}{l}5.2 \% \\
2.3 \% \\
13.3 \% \\
21.9 \%\end{array}$ & $\begin{array}{l}3 \\
0 \\
0 \\
2\end{array}$ & $\begin{array}{l}1.9 \% \\
0 \% \\
0 \% \\
6.3 \%\end{array}$ \\
\hline $\begin{array}{l}\text { This symbol } \\
\text { represents }\end{array}$ & $\begin{array}{l}18-22 \\
23-30 \\
31-39 \\
\text { Greater } \\
\text { than } 40\end{array}$ & $\begin{array}{l}64 \\
16 \\
3 \\
13\end{array}$ & $\begin{array}{l}41.6 \% \\
36.4 \% \\
20 \% \\
40.6 \%\end{array}$ & $\begin{array}{l}71 \\
23 \\
9 \\
15\end{array}$ & $\begin{array}{l}46.1 \% \\
52.3 \% \\
60 \% \\
46.9 \%\end{array}$ & $\begin{array}{l}19 \\
5 \\
3 \\
4\end{array}$ & $\begin{array}{l}12.3 \% \\
11.4 \% \\
20 \% \\
12.5 \%\end{array}$ \\
\hline This & $\begin{array}{l}18-22 \\
23-30 \\
31-39 \\
\text { Greater } \\
\text { than } 40\end{array}$ & $\begin{array}{l}81 \\
14 \\
7 \\
15\end{array}$ & $\begin{array}{l}52.6 \% \\
31.8 \% \\
46.7 \% \\
46.9 \%\end{array}$ & $\begin{array}{l}42 \\
21 \\
6 \\
10\end{array}$ & $\begin{array}{l}27.3 \% \\
47.7 \% \\
40 \% \\
31.3 \%\end{array}$ & $\begin{array}{l}31 \\
9 \\
2 \\
7\end{array}$ & $\begin{array}{l}20.1 \% \\
20.5 \% \\
13.3 \% \\
21.9 \%\end{array}$ \\
\hline $\begin{array}{l}\text { This symbol } \\
\text { represents }\end{array}$ & $\begin{array}{l}18-22 \\
23-30 \\
31-39 \\
\text { Greater } \\
\text { than } 40\end{array}$ & $\begin{array}{l}69 \\
13 \\
6 \\
15\end{array}$ & $\begin{array}{l}44.8 \% \\
29.5 \% \\
40 \% \\
46.9 \%\end{array}$ & $\begin{array}{l}51 \\
12 \\
5 \\
6\end{array}$ & $\begin{array}{l}33.1 \% \\
27.3 \% \\
33.3 \% \\
18.8 \%\end{array}$ & $\begin{array}{l}34 \\
19 \\
4 \\
11\end{array}$ & $\begin{array}{l}22.1 \% \\
43.2 \% \\
26.7 \% \\
34.4 \%\end{array}$ \\
\hline This symbol & $\begin{array}{l}18-22 \\
23-30 \\
31-39 \\
\text { Greater } \\
\text { than } 40\end{array}$ & $\begin{array}{l}83 \\
21 \\
7 \\
16\end{array}$ & $\begin{array}{l}53.9 \% \\
47.7 \% \\
46.7 \% \\
50 \%\end{array}$ & $\begin{array}{l}63 \\
21 \\
8 \\
15\end{array}$ & $\begin{array}{l}40.9 \% \\
47.7 \% \\
53.3 \% \\
46.9 \%\end{array}$ & $\begin{array}{l}8 \\
2 \\
0 \\
1\end{array}$ & $\begin{array}{l}5.2 \% \\
4.5 \% \\
0 \% \\
3.1 \%\end{array}$ \\
\hline $\begin{array}{l}\text { This symbol } \ \\
\text { represents }\end{array}$ & $\begin{array}{l}18-22 \\
23-30 \\
31-39 \\
\text { Greater } \\
\text { than } 40\end{array}$ & $\begin{array}{l}37 \\
13 \\
2 \\
8\end{array}$ & $\begin{array}{l}24 \% \\
29.5 \% \\
13.3 \% \\
25 \%\end{array}$ & $\begin{array}{l}71 \\
21 \\
9 \\
15\end{array}$ & $\begin{array}{l}46.1 \% \\
47.7 \% \\
60 \% \\
46.9 \%\end{array}$ & $\begin{array}{l}46 \\
10 \\
4 \\
9\end{array}$ & $\begin{array}{l}29.9 \% \\
22.7 \% \\
26.7 \% \\
28.1 \%\end{array}$ \\
\hline This & $\begin{array}{l}18-22 \\
23-30 \\
31-39 \\
\text { Greater } \\
\text { than } 40\end{array}$ & $\begin{array}{l}14 \\
2 \\
0 \\
4\end{array}$ & $\begin{array}{l}9.1 \% \\
4.5 \% \\
0 \% \\
12 . \% 5\end{array}$ & $\begin{array}{l}123 \\
35 \\
15 \\
21\end{array}$ & $\begin{array}{l}79.9 \% \\
79.5 \% \\
100 \% \\
65.6 \%\end{array}$ & $\begin{array}{l}17 \\
7 \\
0 \\
7\end{array}$ & $\begin{array}{l}11 \% \\
15.9 \% \\
0 \% \\
21.9 \%\end{array}$ \\
\hline This represents & $\begin{array}{l}18-22 \\
23-30 \\
31-39 \\
\text { Greater } \\
\text { than } 40\end{array}$ & $\begin{array}{l}109 \\
34 \\
10 \\
\\
28\end{array}$ & $\begin{array}{l}70.8 \% \\
77.3 \% \\
66.7 \% \\
87.5 \%\end{array}$ & $\begin{array}{l}17 \\
2 \\
3 \\
1\end{array}$ & $\begin{array}{l}11 \% \\
4.5 \% \\
20 \% \\
3.1 \%\end{array}$ & $\begin{array}{l}28 \\
8 \\
2 \\
\\
3\end{array}$ & $\begin{array}{l}18.2 \% \\
18.2 \% \\
13.3 \% \\
9.4 \%\end{array}$ \\
\hline
\end{tabular}

Table 5. Percentage of correct answers, incorrect answer and not knowing the answer for respondents with respect to education qualification

\begin{tabular}{|l|l|l|l|l|l|l|l|}
\hline \multirow{2}{*}{ questions } & \multirow{2}{*}{$\begin{array}{l}\text { Education } \\
\text { qualification }\end{array}$} & \multicolumn{2}{l|}{ Correct answer } & \multicolumn{2}{l|}{ Incorrect answer } & \multicolumn{2}{l|}{ I don't know } \\
\cline { 3 - 8 } & & number & percent & number & percent & number & percent \\
\hline This symbol & $\begin{array}{l}\text { CURRENTLY } \\
\text { STUDENT }\end{array}$ & 82 & $51.2 \%$ & 51 & $31.9 \%$ & 27 & $16.9 \%$ \\
\hline
\end{tabular}




\begin{tabular}{|c|c|c|c|c|c|c|c|}
\hline represents & $\begin{array}{l}\text { POST GRADUATE } \\
\text { STUDENTS } \\
\text { TEACHERS } \\
\text { LAB } \\
\text { TECHNICIANS } \\
\text { LAB MANEGER } \\
\text { INSTRUCTER } \\
\text { Master OR doctor } \\
\text { OTHERS }\end{array}$ & $\begin{array}{l}8 \\
9 \\
4 \\
9 \\
6\end{array}$ & $\begin{array}{l}42.1 \% \\
\\
57.1 \% \\
75 \% \\
66.7 \% \\
60 \% \\
31.6 \%\end{array}$ & $\begin{array}{l}1 \\
1 \\
0 \\
2 \\
8\end{array}$ & $\begin{array}{l}36.8 \% \\
\\
7.1 \% \\
8.3 \% \\
\\
0 \% \\
13.3 \% \\
42.1 \%\end{array}$ & $\begin{array}{l}5 \\
2 \\
\\
2 \\
4 \\
5\end{array}$ & $\begin{array}{l}35.7 \% \\
16.7 \% \\
33.3 \% \\
26.7 \% \\
26.3 \%\end{array}$ \\
\hline This symbol & $\begin{array}{l}\text { CURRENTLY } \\
\text { STUDENT } \\
\text { POST GRADUATE } \\
\text { STUDENTS } \\
\text { TEACHERS } \\
\text { LAB } \\
\text { TECHNICIANS } \\
\text { LAB MANEGER } \\
\text { INSTRUCTER } \\
\text { Master OR doctor } \\
\text { OTHERS }\end{array}$ & $\begin{array}{l}13 \\
12 \\
3 \\
12 \\
16\end{array}$ & $\begin{array}{l}93.8 \% \\
84.2 \% \\
\\
92.9 \% \\
100 \% \\
\\
50 \% \\
80 \% \\
84.2 \%\end{array}$ & $\begin{array}{l}1 \\
0 \\
\\
3 \\
1 \\
3\end{array}$ & $\begin{array}{l}4.4 \% \\
15.8 \% \\
7.1 \% \\
0 \% \\
50 \% \\
6.7 \% \\
15.8 \%\end{array}$ & $\begin{array}{l}0 \\
0 \\
0 \\
2 \\
0\end{array}$ & $\begin{array}{l}0 \% \\
0 \% \\
0 \% \\
13.3 \% \\
0 \%\end{array}$ \\
\hline $\begin{array}{l}\text { This symbol } \\
\text { represents }\end{array}$ & $\begin{array}{l}\text { CURRENTLY } \\
\text { STUDENT } \\
\text { POST GRADUATE } \\
\text { STUDENTS } \\
\text { TEACHERS } \\
\text { LAB } \\
\text { TECHNICIANS } \\
\text { LAB MANEGER } \\
\text { INSTRUCTER } \\
\text { Master OR doctor } \\
\text { OTHERS }\end{array}$ & $\begin{array}{l}6 \\
5 \\
\\
2 \\
4 \\
5\end{array}$ & $\begin{array}{l}43.8 \% \\
21.1 \% \\
\\
42.9 \% \\
41.7 \% \\
\\
33.3 \% \\
26.7 \% \\
26.3 \%\end{array}$ & $\begin{array}{l}8 \\
7 \\
4 \\
5 \\
12\end{array}$ & $\begin{array}{l}43.1 \% \\
68.4 \% \\
57.1 \% \\
58.3 \% \\
\\
66.7 \% \\
33.3 \% \\
63.2 \%\end{array}$ & $\begin{array}{l}0 \\
0 \\
0 \\
6 \\
2\end{array}$ & $\begin{array}{l}13.1 \% \\
10.5 \% \\
0 \% \\
0 \% \\
\\
0 \% \\
40 \% \\
10.5 \%\end{array}$ \\
\hline This & $\begin{array}{l}\text { CURRENTLY } \\
\text { STUDENT } \\
\text { POST GRADUATE } \\
\text { STUDENTS } \\
\text { TEACHERS } \\
\text { LAB } \\
\text { TECHNICIANS } \\
\text { LAB MANEGER } \\
\text { INSTRUCTER } \\
\text { Master OR doctor } \\
\text { OTHERS }\end{array}$ & $\begin{array}{l}6 \\
5 \\
4 \\
6 \\
6\end{array}$ & $\begin{array}{l}42.9 \% \\
41.7 \% \\
\\
66.7 \% \\
40 \% \\
31.6 \%\end{array}$ & $\begin{array}{l}7 \\
6 \\
\\
2 \\
3 \\
11\end{array}$ & $\begin{array}{l}25 \% \\
52.6 \% \\
50 \% \\
50 \% \\
\\
33.3 \% \\
20 \% \\
57.9 \%\end{array}$ & $\begin{array}{l}1 \\
1 \\
0 \\
6 \\
2\end{array}$ & $\begin{array}{l}7.1 \% \\
8.3 \% \\
\\
0 \% \\
40 \% \\
10.5 \%\end{array}$ \\
\hline $\begin{array}{l}\text { This symbol } \\
\text { represents }\end{array}$ & $\begin{array}{l}\text { CURRENTLY } \\
\text { STUDENT } \\
\text { POST GRADUATE } \\
\text { STUDENTS } \\
\text { TEACHERS } \\
\text { LAB } \\
\text { TECHNICIANS } \\
\text { LAB MANEGER } \\
\text { INSTRUCTER }\end{array}$ & $\begin{array}{l}70 \\
7\end{array}$ & $\begin{array}{l}43.8 \% \\
36.8 \% \\
42.9 \% \\
58.3 \%\end{array}$ & $\begin{array}{l}51 \\
6\end{array}$ & $\begin{array}{l}31.9 \% \\
31.6 \%\end{array}$ & $\begin{array}{l}39 \\
6\end{array}$ & $\begin{array}{l}24.4 \% \\
31.6 \%\end{array}$ \\
\hline
\end{tabular}




\begin{tabular}{|c|c|c|c|c|c|c|c|}
\hline & $\begin{array}{l}\text { Master OR doctor } \\
\text { OTHERS }\end{array}$ & $\begin{array}{l}3 \\
6 \\
4\end{array}$ & $\begin{array}{l}50 \% \\
40 \% \\
21.1\end{array}$ & $\begin{array}{l}2 \\
2 \\
9\end{array}$ & $\begin{array}{l}33.3 \% \\
13.3 \% \\
47.4\end{array}$ & $\begin{array}{l}1 \\
7 \\
6\end{array}$ & $\begin{array}{l}16.7 \% \\
46.7 \% \\
31.6 \%\end{array}$ \\
\hline \multirow{7}{*}{ This } & $\begin{array}{l}\text { CURRENTLY } \\
\text { STUDENT }\end{array}$ & 93 & $58.1 \%$ & 58 & $36.3 \%$ & 9 & $5.6 \%$ \\
\hline & $\begin{array}{l}\text { POST GRADUATE } \\
\text { STUDENTS } \\
\text { TEACHERS }\end{array}$ & 7 & $36.8 \%$ & 12 & $63.2 \%$ & 0 & $0 \%$ \\
\hline & LAB & 8 & $57.1 \%$ & 6 & $42.9 \%$ & 0 & $0 \%$ \\
\hline & $\begin{array}{l}\text { TECHNICIANS } \\
\text { LAB MANEGER }\end{array}$ & 5 & $41.7 \%$ & 7 & $58.3 \%$ & 0 & $0 \%$ \\
\hline & INSTRUCTER & 3 & $50 \%$ & 3 & $50 \%$ & 0 & $0 \%$ \\
\hline & Master OR doctor & 4 & $26.7 \%$ & 9 & $60 \%$ & 2 & $13.3 \%$ \\
\hline & OTHERS & 7 & $36.8 \%$ & 12 & $63.2 \%$ & 0 & $0 \%$ \\
\hline \multirow{8}{*}{$\begin{array}{l}\text { This symbol }\langle\mathbf{\imath} \\
\text { represents }\end{array}$} & $\begin{array}{l}\text { CURRENTLY } \\
\text { STUDENT }\end{array}$ & 35 & $21.9 \%$ & 74 & $46.3 \%$ & 51 & $31.9 \%$ \\
\hline & POST GRADUATE & 6 & $31.6 \%$ & 10 & $52.6 \%$ & 3 & $15.8 \%$ \\
\hline & TEACHERS & & & & & & \\
\hline & LAB & 5 & $35.7 \%$ & 7 & $50 \%$ & 2 & $14.3 \%$ \\
\hline & $\begin{array}{l}\text { TECHNICIANS } \\
\text { LAB MANEGER }\end{array}$ & 7 & $58.3 \%$ & 4 & $33.3 \%$ & 1 & $8.3 \%$ \\
\hline & INSTRUCTER & 1 & $16.7 \%$ & 2 & $33.3 \%$ & 3 & $50 \%$ \\
\hline & Master OR doctor & 3 & $20 \%$ & 7 & $46.7 \%$ & 5 & $33.3 \%$ \\
\hline & OTHERS & 3 & $15.8 \%$ & 12 & $63.2 \%$ & 4 & $21.1 \%$ \\
\hline \multirow{8}{*}{ This represents } & $\begin{array}{l}\text { CURRENTLY } \\
\text { STUDENT }\end{array}$ & 13 & $8.1 \%$ & 128 & $80 \%$ & 19 & $11.9 \%$ \\
\hline & $\begin{array}{l}\text { POST GRADUATE } \\
\text { STUDENTS }\end{array}$ & 2 & $10.5 \%$ & 14 & $73.7 \%$ & 3 & $15.8 \%$ \\
\hline & TEACHERS & & & & & & \\
\hline & LAB & 1 & $7.1 \%$ & 13 & $92.9 \%$ & 0 & $0 \%$ \\
\hline & $\begin{array}{l}\text { TECHNICIANS } \\
\text { LAB MANEGER }\end{array}$ & 2 & $16.7 \%$ & 10 & $83.3 \%$ & 0 & $0 \%$ \\
\hline & INSTRUCTER & 1 & $16.7 \%$ & 2 & $33.3 \%$ & 3 & $50 \%$ \\
\hline & Master OR doctor & 0 & $0 \%$ & 11 & $73.3 \%$ & 4 & $26.7 \%$ \\
\hline & OTHERS & 1 & $5.3 \%$ & 16 & $84.2 \%$ & 2 & $10.5 \%$ \\
\hline \multirow{8}{*}{$\begin{array}{l}\text { This } \\
\text { represents }\end{array}$} & $\begin{array}{l}\text { CURRENTLY } \\
\text { STUDENT }\end{array}$ & 110 & $68.8 \%$ & 17 & $10.6 \%$ & 33 & $20.6 \%$ \\
\hline & $\begin{array}{l}\text { POST GRADUATE } \\
\text { STUDENTS }\end{array}$ & 17 & $89.5 \%$ & 1 & $5.3 \%$ & 1 & $5.3 \%$ \\
\hline & TEACHERS & & & & & & \\
\hline & LAB & 12 & $\begin{array}{l}85.7 \% \\
100 \%\end{array}$ & 2 & $14.3 \%$ & 0 & $0 \%$ \\
\hline & LAB MANEGER & 12 & $100 \%$ & 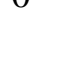 & & 0 & \\
\hline & INSTRUCTER & 6 & $100 \%$ & 0 & $0 \%$ & 0 & $0 \%$ \\
\hline & Master OR doctor & 10 & $66.7 \%$ & 1 & $6.7 \%$ & 4 & $26 . \% 7$ \\
\hline & OTHERS & 14 & $73.7 \%$ & 2 & $10.5 \%$ & 3 & $15.8 \%$ \\
\hline
\end{tabular}


Contribution of Individual Authors to the Creation of a Scientific Article (Ghostwriting Policy)

Nawal H Bahtiti: Made a significant contribution to the work reported.

Ahmad Abu Rayyan: Have drafted or written, substantially revised or critically reviewed the article

Tala Sasa: Study design, execution, acquisition of data, analysis and interpretation

Waed al Ahmad: Reviewed and agreed on all versions of the article before submission, during revision, the final version accepted for publication, and any significant changes introduced at the proofing stage.

Sources of Funding for Research Presented in a Scientific Article or Scientific Article Itself

There are no sources of funds

Creative Commons Attribution License 4.0 (Attribution 4.0 International, CC BY 4.0)

This article is published under the terms of the Creative Commons Attribution License 4.0

https://creativecommons.org/licenses/by/4.0/deed.en US1- 